\title{
Étude de la prévalence des maladies liées à l'eau et influences des facteurs environnementaux dans l'arrondissement de Nomgr-Masson : cas du quartier Tanghin (Ouagadougou-Burkina Faso)
}

\author{
Yelezouomin Stéphane Corentin SOMÉ ${ }^{1 *}$, Thomas Domegnon SORO ${ }^{1}$ et \\ Souleymane OUEDRAOGO ${ }^{2}$ \\ ${ }^{1}$ International Institute for Water and Environmental Engineering; Laboratory of Hydrology and Water \\ Resources (Foundation 2iE), Burkina Faso. \\ ${ }^{2}$ Direction Générale de l'Aménagement du Territoire, Ministère de l'Economie et de Finance, Burkina Faso. \\ *Corresponding author; E-mail: some_y@yahoo.fr; corentin.some@2ie-edu.org,
}

\section{RESUME}

Les maladies liées à l'eau font partie des maladies infectieuses les plus préoccupantes observées au sein des populations des pays en développement. L'objectif de ce travail est d'étudier la contribution des maladies liées à l'eau aux dix principales causes de consultations à Tanghin et les facteurs de risques comportementaux et environnementaux qui y contribuent. La méthodologie s'articule autour d'une enquête géographique, de l'analyse microbiologique et physico-chimique des eaux et d'une enquête ménage. Les maladies liées à l'eau occupent plus de $40 \%$ des dix principales causes de consultation à Tanghin. Le paludisme est la maladie la plus répandue, suivie de la fièvre typhoïde et la dysenterie. Les eaux de puits sont toutes contaminées par les coliformes totaux et thermotolérants. Le niveau d'assainissement du quartier est très bas et permet d'observer de nombreux dépotoirs sauvages même aux abords de point d'eau. La gestion de l'eau de consommation n'est également pas convenable. Cela a pour effet une forte exposition de la population aux risques sanitaires liés à l'eau. 9.2\% de la population estime avoir été infectés par les maladies liées à l'eau au cours de l'année. Une action urgente en matière d'assainissement et gestion de l'eau dans les ménages est donc à entreprendre.

(c) 2014 International Formulae Group. All rights reserved.

Mots clés : maladies liées à l'eau, pollution microbiologique, assainissement, Tanghin, Burkina Faso.

\section{INTRODUCTION}

Par « maladies liées à l'eau », il faut comprendre, maladies contractées par ingestion, par contact direct ou encore les maladies pour lesquelles l'eau est un habitat d'hôtes de larves ou de parasites. Ces maladies sont nombreuses et ont fait l'objet de plusieurs classifications (Resh, 2009; Diakité et al., 2010 ; Kpoda et al., 2013; Ouledi et al.,
2012). On distingue globalement 6 types de maladies liées à l'eau :

- les maladies hydriques ou maladies de l'eau sale. Ce sont des maladies qui proviennent de la pollution humaine (péril fécal) et de la pollution chimique ;

- Les maladies à support hydrique ou maladie aquatique. Dans ce cas, l'eau est un habitat 
utilisé par des hôtes passifs qui servent de réservoir de pathogène ;

- Les maladies dues aux vecteurs liés à l'eau. Dans ce cas, le milieu aquatique est un milieu dans lequel les vecteurs de maladies passent au moins une partie de leur cycle de vie

- les maladies aggravées par la pénurie.

D'après OMS et l'UNICEF «les maladies liées à l'eau sont une tragédie humaine dont le poids se fait de plus en plus lourd, tuant plus de 5 millions de gens chaque année - 10 fois le nombre de personnes tuées par les guerres. Environ 2,3 milliards de gens souffrent de maladies dues à une mauvaise qualité de l'eau. Environ $60 \%$ de la mortalité infantile dans le monde est due à des maladies infectieuses ou parasitaires, la plupart liées à l'eau. » Pour Global Health and Education Foundation, les populations les plus pauvres des pays en développement, particulièrement les enfants, en souffrent le plus. Les maladies liées à l'eau maintiennent des millions de personnes dans un cercle vicieux de pauvreté et de mauvaise santé, les rendant ainsi incapables de travailler ou d'aller à l'école. Dans le monde en développement, les effets cumulés des maladies liées à l'eau paralysent l'économie et pèsent lourdement sur des organismes médicaux déjà surchargés. »

En outre, la mauvaise qualité de l'eau, l'hygiène défectueuse et l'insuffisance d'assainissement sont à l'origine des maladies liées à l'eau notamment les maladies hydriques (Ledeur, 2004; Kientga, 2008). Chaque année, près de 8 millions de personnes, dont la moitié sont des enfants, meurent de maladies dues à la qualité ou à l'absence de l'eau, d'hygiène ou d'assainissement (Camdessus et al., 2004).

Les eaux de surface jouent un rôle important dans la répartition des vecteurs et la transmission spatiale et temporelle des maladies (Poda, 2007). 80\% des maladies et plus d'un tiers des décès dans les pays en développement sont dus à la consommation d'eau de mauvaise qualité (Kombasséré, 2007). Situé dans l'arrondissement de NongrMasson, Tanghin est un quartier périphérique de la ville de Ouagadougou. C'est un quartier qui connaît d'énormes problèmes de santé et d'assainissement (DEP, 2008). Un aperçu des consultations en décembre 2008 montre que sur les dix maladies qui sont les principales causes de consultations dans le district sanitaire de Kossodo qui couvre géographiquement l'arrondissement de Nongr-Masson, dont relève ce quartier, neuf sont des maladies liées à l'eau (DEP, 2010).

Voilà une synthèse peu enviable qui caractérise la situation de l'arrondissement de Nongr-Masson dans le domaine de l'assainissement et de la santé. Qu'en est-il du quartier Tanghin? L'objectif de ce travail est d'évaluer la contribution des maladies liées à l'eau à Tanghin. Ce travail s'inscrit dans le cadre d'un projet de recherche en Ecosanté qui vise à contribuer à une réflexion globale en vue d'une solution durable aux problèmes de santé liés à l'environnement.

\section{MATERIEL ET METHODES}

Relevant de l'arrondissement de Nongr-Masson de la commune de Ouagadougou, Tanghin (secteurs 23 et secteur 24) est un quartier traditionnel partiellement aménagé (Figure 1). Sa superficie de 1765,6 ha avec 307,4 ha pour le secteur 24 et 1458.2 ha pour le secteur 23. Sa population est composée d'autochtone mossis, de migrants anciens Peulh éleveurs nomades sédentarisés et de nouveaux migrants (Sabatinelli, 1989). Sa population est de 54247 habitants (INSD, 1996) et de 81838 habitants en 2006 (INSD, 2006). Le quartier Tanghin est riverain du barrage $\mathrm{N}^{\circ} 2$ de Ouagadougou par le Sud, à l'Est par les secteurs 25 et 13 et à l'Ouest par le secteur 22 .

Les secteurs 23 et 24 de l'arrondissement de Nongr-Masson, qui constituent Tanghin ont été choisis comme site pour notre étude.

\section{Le choix du site}

Le choix du quartier Tanghin est motivé par plusieurs facteurs que sont la présence d'un plan d'eau en l'occurrence les barrages $\mathrm{N}^{\circ} 2$ et $\mathrm{N}^{\circ} 3$ de Ouagadougou, les 
diversités du niveau d'aménagement, la diversité socioculturelle des populations et la diversité des sources d'eau. Cette configuration géographique et sociale nous a semblé originale et bien adaptée à une telle étude. De plus, notre travail profite de la présence d'associations œuvrant dans le domaine de l'environnement et de l'assainissement qui a facilité l'administration du questionnaire et la participation effective de la population au projet. En effet, ce travail s'est déroulé dans le cadre d'un projet de recherche en écosanté qui par essence est transdisciplinaire, systémique et participatif et qui met en œuvre une nouvelle approche des questions de santé.

\section{Approche méthodologique générale}

L'approche méthodologique générale s'est articulée autour de quatre phases (Figure 2) qui sont ci-dessous détaillées.

\section{Phase préliminaire}

Elle a consisté en l'élaboration du cadre logique du projet afin de fixer les objectifs à atteindre.

\section{Phase de terrain}

La phase de terrain a consisté à :

$\checkmark$ relever les coordonnées géographiques des points d'eau potable, points de rejets des eaux usées et des dépotoirs de déchets solides à l'aide d'un GPS ;

$\checkmark$ faire une enquête ménage afin d'établir la structure (en terme de fréquence) des maladies liées à l'eau et d'appréhender les facteurs de risque liés à l'environnement ;

$\checkmark$ collecter les données sanitaires à la direction des études et de la planification (DEP) du Ministère de la santé ;

$\checkmark$ faire des prélèvements des eaux de puits et des eaux de boisson stockées dans les ménages en vue des analyses physicochimiques et microbiologiques.

\section{Phase d'analyse au laboratoire}

Elle avait pour objectif de vérifier la qualité des eaux de puits, et les eaux de boisson stockées par les ménages et ainsi que celles des sources (forages, bornes fontaines, postes d'eau). Les coliformes et streptocoques fécaux ont été recherchés quantitativement.

\section{Phase de traitement des données}

La phase de traitement des données a consisté au traitement des données collectées sur les logiciels tels qu'EpiInfo, Arcview3.2a et à l'établissement des cartes.

Sur le plan des méthodes, des enquêtes géographiques, des enquêtes ménages et des analyses physico-chimiques et microbiologiques des eaux ont été effectuées. Une analyse statistique et une analyse spatiale des données ont été effectuées sur les résultats obtenus.

L'enquête géographique a consisté en la collecte des coordonnées géographiques de tous les points d'eau communautaires (forages, puits, borne fontaine, poste d'eau autonome), les décharges sauvages de déchets solides et de point de rejet des eaux usées. L'objectif de cette enquête géographique est de servir de base pour l'échantillonnage de l'eau à analyser, de fournir une base d'analyse et interprétation des résultats.

L'enquête ménage est une enquête d'administration directe par questionnaire sur un échantillon de 200 ménages tiré de façon aléatoire. La population cible est constituée des chefs de ménage. L'objectif de l'enquête a été de produire de données primaires sur les maladies et leurs fréquences au sein des ménages et les comportements des ménages en matière d'assainissements et de gestion des eaux; ceci en appui à la consultation des statistiques sanitaire du district sanitaire de Kossodo. Les données collectées mises en relation avec les résultats de la consultation des statistiques sanitaires devraient conduire à une interprétation plus profonde du profil épidémiologique de Tanghin.

L'analyse microbiologique et physicochimique des eaux a concerné 300 échantillons d'eau repartie entre les eaux de consommation à domicile, les points d'eau communautaire (forages, bornes fontaines, poste d'eau autonome et puits).

Les paramètres physiques tels que la température, le $\mathrm{pH}$, la conductivité, la turbidité et les solides totaux dissous ont été mesurés in situ. Pour la température, la conductivité et les solides totaux dissous, les 
mesures ont été effectué à l'aide d'un multiparamètre de type $\mathrm{HACH}$; pour la turbidité, c'est un photomètre marque Palintest 7500 qui a été utilisé. Le pH a été mesuré à l'aide d'un pH-mètre de type WTW $330 \mathrm{i}$.

En ce qui concerne les paramètres microbiologiques, les éléments recherchés sont les indicateurs de contaminations fécales (coliformes totaux, coliformes fécaux, E. coli) et les streptocoques fécaux. La méthode d'ensemencement sur milieu solide après filtration sur membrane a été utilisée (RODIER, 1996; SEMT, 2006). Le milieu utilisé pour la culture des coliformes totaux et des coliformes fécaux est le milieu chromocult. Celui des streptocoques fécaux est la gélose Slanetz et Bartley + TTC.
L'incubation est faite à $37{ }^{\circ} \mathrm{C}$ pour les coliformes totaux et les streptocoques fécaux et à $44{ }^{\circ} \mathrm{C}$ pour les coliformes fécaux. La durée de l'incubation est de $24 \mathrm{~h}$ pour les coliformes et de $48 \mathrm{~h}$ pour les streptocoques fécaux. Après incubation, la présence de coliformes est marquée par l'apparition de colonies de différentes couleurs.

Les résultats des différents travaux ont fait l'objet de discussion lors d'un atelier organisé à la Mairie de l'arrondissement Nongr-Masson, de commun accord avec les autorités communales, administratives et l'équipe de chercheurs du LEDES (Laboratoire Eau Dépollution Ecosystème et Santé) du Centre Commun de recherche Eau et Climat de la Fondation 2iE.

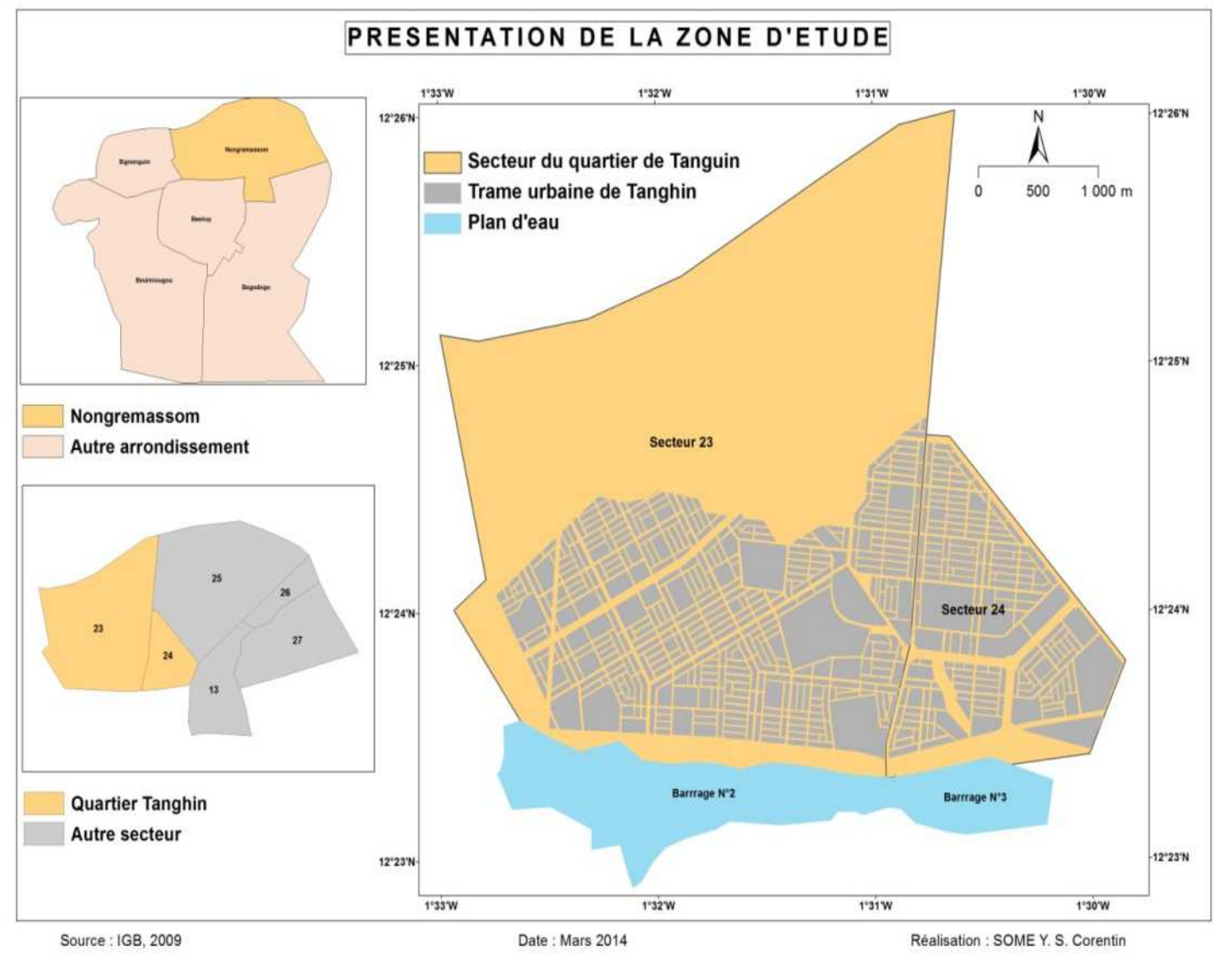

Figure1 : Situation de la zone d'étude. 


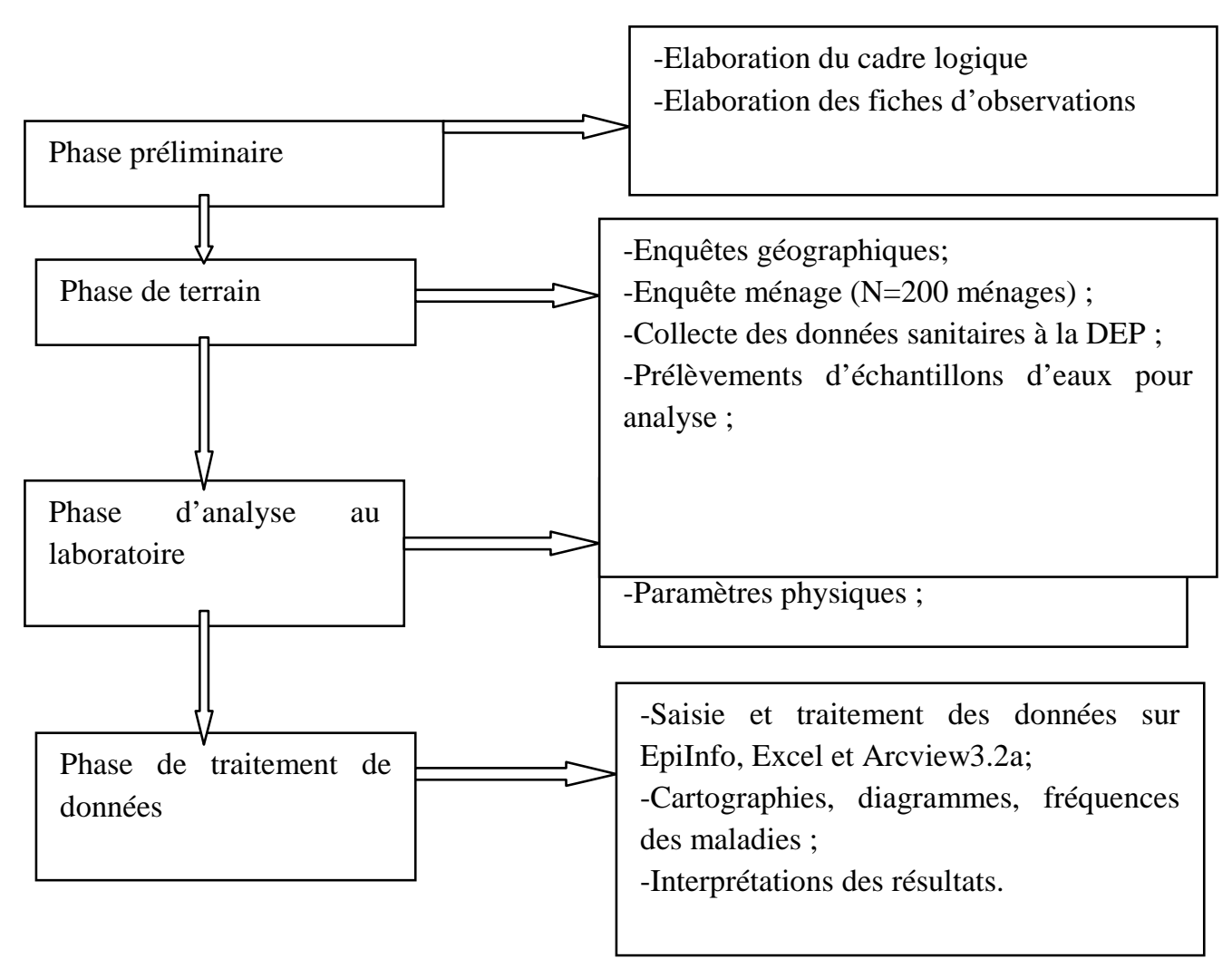

Figure 2 : Présentation de l'approche méthodologique générale.

\section{RESULTATS}

Fréquence des dix principales maladies de l'arrondissement

Il ressort de l'analyse des différents plans d'action du district de Kossodo, que seuls les résultats des statistiques de morbidités des 3 dernières années (2007, 2009 et 2010) sont disponibles. Etant donné que les statistiques sanitaires des registres de Tanghin étaient inaccessibles, nous avons dû utiliser ceux du District sanitaire de Kossodo comme bases de données pour cette étude.

\section{Poids des maladies liées à l'eau}

Selon le Plan d'action du District de Kossodo, les maladies liées à l'eau représentent respectivement $41 \%$ en 2007 , $46 \%$ en 2009 et $49 \%$ en 2010 du taux de morbidité total des 10 principales causes de consultations des ménages. Ces dix principales causes de consultations représentent plus de $80 \%$ de l'ensemble des motifs de consultations. Les maladies liées à l'eau constituent les plus importants motifs des consultations dans les différents CSPS de l'arrondissement de Nongr-Masson.

On constate qu'environ $50 \%$ des consultations des registres sanitaires du district sont des maladies liées à l'eau (eaux usées, eaux impropres à la consommation, manque d'eau....) pour l'année 2010. Ce qui traduit l'implication de l'eau dans les différents problèmes majeurs de santé publique de l'arrondissement. Les résultats des années 2007, 2009 et 2010 montrent une croissance de la part des maladies liées à l'eau dans la morbidité du district.

Le Tableau 2 montre la qualité bactériologique des eaux de puits utilisées par les ménages comme source de boisson et de bain. On observe que la qualité de l'eau de puits est mauvaise tant pour la boisson que pour les besoins corporels. Aucun puits n'est exempt de contamination bactérienne. 


\section{Qualité des eaux de boisson stockées}

L'eau considérée comme potable à la sortie de la borne fontaine, du forage, du poste d'eau et du robinet est soumise aux différents risques de contamination que sont les pratiques qu'adoptent les ménages lors de la collecte d'une part et les moyens de collecte et de conservation de l'eau d'autre part. Les résultats de l'analyses des eaux de consommation stockées dans les ménages montrent la modification de la qualité de l'eau depuis la source jusqu'à la consommation. On remarque que la charge bactérienne des coliformes totaux augmente depuis la source (parfois nulle 0 ufc/ml) jusqu'à 1006 ufc/100 ml. Cela traduit une contamination de l'eau dans la chaîne de l'eau. Cette contamination fait courir aux ménages un risque sanitaire lié à l'usage l'eau.

\section{Facteurs de risques physiques et} comportementaux liées aux maladies

La qualité des eaux dans les ménages dépend de plusieurs facteurs dont deux nous semblent les plus importants. La première dépend de la source et partant du mode d'approvisionnement et de la gestion de l'eau qui intègre des éléments telle l'hygiène dans le stockage de l'eau et la seconde, de la durée de stockage des eaux.

\section{Modes d'approvisionnement en eau potable des ménages}

Les diagrammes présentent des résultats pratiques des ménages. On observe que certains ménages s'approvisionnent en une seule source à savoir, à $46 \%$ à la borne fontaine, à $37 \%$ au branchement privé, à $3 \%$ au puits et à $1 \%$ au poste d'eau (Figure 7). D'autres ménages s'approvisionnent en deux sources telles que les puits et les branchements privés $(6 \%)$, les puits et les bornes fontaines $(5 \%)$, les forages et les bornes fontaines (1\%), les forages et branchements privés (1\%). Les bornes fontaines et les branchements privés sont les sources les plus utilisés par les ménages de Tanghin pour l'approvisionnement en eau potable. La Figure 3 montre que le taux de morbidité lié à l'eau est de $41 \%$ plus du tiers du taux de morbidité totale de la population. En 2009, la part des maladies liées à l'eau a augmenté (Figure 4).

- Cas de l'année 2009

La Figure 4 montre que le taux de morbidité lié à l'eau est de $44 \%$ plus du tiers du taux de morbidité totale de la population.

- Cas de l'année 2010

La Figure 5 nous montre que le taux de morbidité liée à l'eau est d'environ $50 \%$ voir la moitié du taux de morbidité totale de la population.

On observe une augmentation du taux de morbidité liée à l'eau au cours des années, passant de $41 \%$ en 2007 à $49 \%$ en 2010. Le maintien d'une telle tendance va conduire à augmentation de la nocivité de l'eau qui est pourtant indispensable à la vie.

Profil épidémiologique des maladies liées à l'eau dans le quartier Tanghin

Fréquences des maladies liées à l'eau : cas de morbidités

L'affection au paludisme a été la maladie la plus répandue, suivie par la diarrhée, la fièvre typhoïde et la dysenterie.

Parmi toutes les personnes dans l'échantillon de la population $(n=1384), 9.2 \%$ $(\mathrm{n}=128)$ ont été trouvés infectés (Tableau 1). $\mathrm{n}=128$ est le nombre de cas de maladies déclarées depuis le début d'année pendant l'enquête.

\section{Taux de prévalences des maladies liées à l'eau}

Les résultats des enquêtes présentent les différentes proportions de prévalence des maladies liées à l'eau. Ainsi, on remarque que le paludisme représente la plus grande prévalence $(8.7 \%)$ parmi les différentes affections. La tranche d'âge des enfants de moins de cinq ans et les femmes présentent les plus importantes prévalences palustres.

$\mathrm{Au}$ niveau de l'affection diarrhéique, elle se présente en deuxième place avec une prévalence de $0.36 \%$. La couche d'enfant de moins d'un an est la plus vulnérable dont la prévalence est de $1.88 \%$. 
En termes de typologie, on remarque que les maladies hydriques prédominent dans le quartier Tanghin. Les maladies transmises par des vecteurs liés à l'eau quant à elles présentent un problème majeur de santé publique de part leur ampleur (fréquence de morbidités).

Le profil épidémiologique des maladies liées à l'eau est dominé:

* selon le type de maladies par les maladies hydriques. Leur émergence se traduit par l'ingestion de l'eau contaminée comme le cas de l'eau des puits investigués ;

* selon l'ampleur par les maladies transmises par les vecteurs liés à l'eau, du fait de la mauvaise gestion de l'eau et de l'environnement.

Modes de gestion des eaux de consommation dans les ménages

D'après les résultats des enquêtes, la plupart des ménages ont adopté des comportements de premier niveau favorables au maintien de la qualité des eaux de consomation (Figure 8 et 9). En effet, 97\% des ménages couvrent les récipients contenant l'eau et $71 \%$ renouvellent journalièrement les stockes d'eaux. Si cela peut réjouir, d'autres situations sont de nature à entamer la qualité de l'eau. Il s'agit par exemple de mode de prélèvement de l'eau stockée, l'hygiène des usagers de l'eau dans le ménage, etc.
Facteurs de risques environnementaux liés aux maladies

Les eaux usées

La cartographie des eaux usées (Figure 10) permet de se rendre à l'évidence une gestion insatisfaisante des eaux usées dans les ménages. Ces rejets sauvages d'eau usée peuvent servir de de milieu de reproduction de certains vecteurs de maladies.

On a pu dénombrer 168 points de rejets d'eaux usées ou d'eaux stagnantes dans le quartier Tanghin. C'est une densité de 0,1 rejet par hectare. Ces eaux sont rejetées dans la rue, qui représente avec une telle densité, une situation d'insalubrité du quartier.

\section{Les déchets solides}

Les décharges sauvages de déchets solides impactent la propagation de certaines maladies liées à l'eau. En effet, ils constituent des sources de contamination des eaux en constituant des réservoirs de pathogènes. La Figure 11, présente une cartographie des décharges sauvages dans le quartier Tanghin.

On a pu dénombrer 510 points de dépotoirs sauvages dans le quartier Tanghin, soit une densité de 0,3 décharge par hectare. Ces dépotoirs de déchets sont répandus sur la voie publique et/ou souvent à des carrefours.

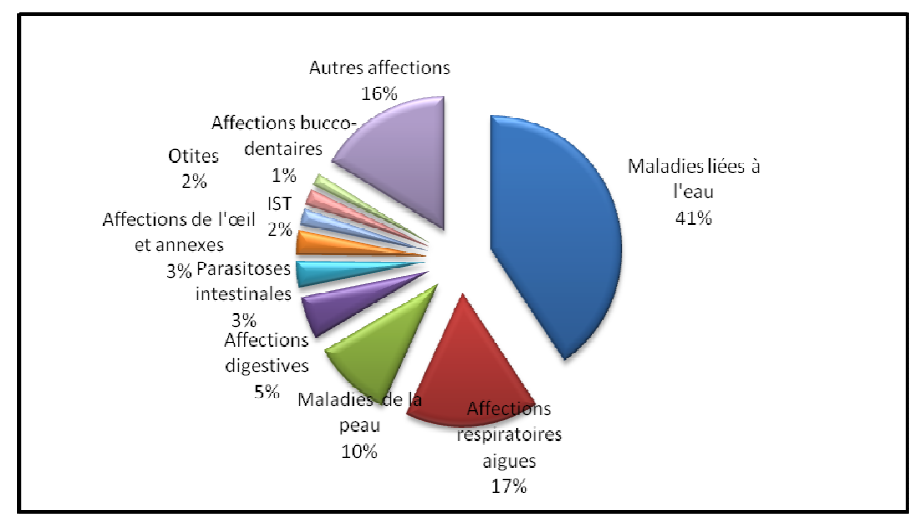

Figure 3: Taux de morbidité des dix principales affections pour l'année 2007. 


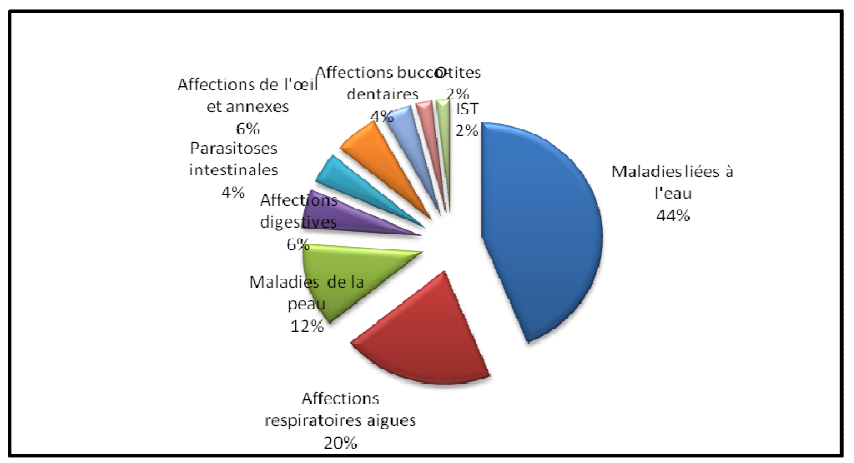

Figure 4:Taux de morbidité des 10 principales affections pour l'année 2009.

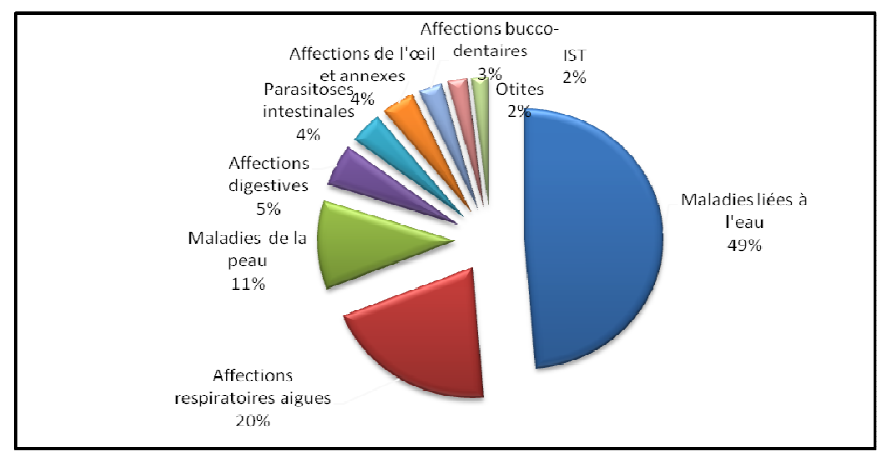

Figure 5:Taux de morbidité des 10 principales affections pour l'année 2010.

Tableau 1: Fréquence des maladies liées à l'eau dans le quartier Tanghin, Ouagadougou.

\begin{tabular}{lcc}
\hline Affections & Nombre de cas & Fréquence(\%) \\
\hline Paludisme & 121 & 94 \\
Diarrhées & 5 & 4 \\
Fièvre typhoïde & 1 & 1 \\
Dysenterie & 1 & 1 \\
Total & $\mathrm{n}=128$ & 100 \\
\hline
\end{tabular}

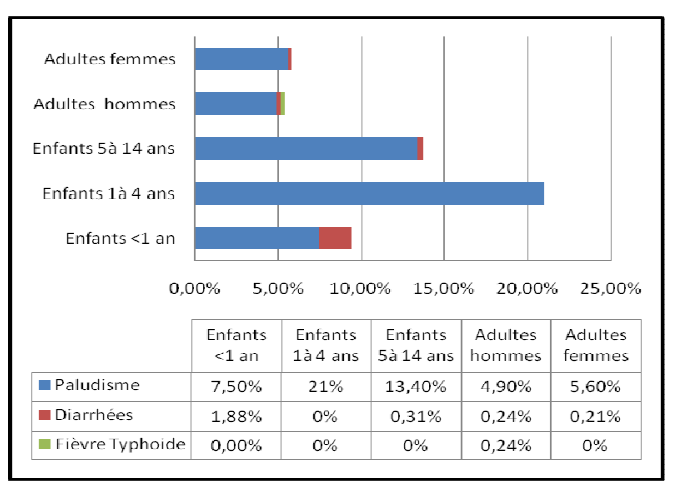

Figure 6: Prévalences des maladies liées à l'eau dans le quartier Tanghin. 
Tableau 2: Résultats des analyses bactériologiques des eaux de puits de Tanghin.

\begin{tabular}{|c|c|c|c|c|}
\hline $\mathbf{N}^{\circ}$ du puits & $\begin{array}{c}\text { Coliformes totaux } \\
(\text { ufc/100 ml })\end{array}$ & $\begin{array}{c}\text { Coliformes fécaux } \\
(\text { ufc/100 ml })\end{array}$ & $\begin{array}{c}\text { E. coli (ufc/100 } \\
\text { ml) }\end{array}$ & $\begin{array}{c}\text { Streptocoques fécaux } \\
(\text { ufc/100 ml })\end{array}$ \\
\hline $1 *$ & 800 & 224 & 416 & 0 \\
\hline $2 *$ & 384 & 304 & 48 & 0 \\
\hline $3^{*}$ & 608 & 592 & 176 & 0 \\
\hline $4^{*}$ & 672 & 256 & 56 & 0 \\
\hline $5 *$ & 1240 & 560 & 160 & 0 \\
\hline $6^{*}$ & 624 & 356 & 36 & 0 \\
\hline $7 *$ & 676 & 608 & 480 & 0 \\
\hline $8^{*}$ & 288 & 32 & 255 & 0 \\
\hline $9 *$ & 1746 & 1404 & 294 & 0 \\
\hline $10 *$ & 640 & 576 & 96 & 0 \\
\hline 11 & 384 & 304 & 360 & 0 \\
\hline $12 *$ & 768 & 416 & 384 & 0 \\
\hline $13 *$ & 516 & 384 & 144 & 0 \\
\hline $14 *$ & 544 & 360 & 448 & 0 \\
\hline 15 & 544 & 224 & 160 & 0 \\
\hline $16^{*}$ & 640 & 480 & 60 & 0 \\
\hline $17 *$ & 648 & 640 & 480 & 0 \\
\hline $18 *$ & 600 & 596 & 80 & 0 \\
\hline $19 *$ & 540 & 440 & 260 & 0 \\
\hline $20 *$ & 304 & 224 & 32 & 0 \\
\hline $21 *$ & 180 & 160 & 20 & 0 \\
\hline $22 *$ & 720 & 368 & 400 & 0 \\
\hline 23 & 224 & 224 & 64 & 0 \\
\hline $24 *$ & 436 & 432 & 208 & 0 \\
\hline 25 & 368 & 272 & 208 & 0 \\
\hline $26 *$ & 648 & 316 & 160 & 0 \\
\hline 27 & 448 & 432 & 240 & 0 \\
\hline $28 *$ & 640 & 320 & 320 & 0 \\
\hline $29 *$ & 800 & 692 & 192 & 0 \\
\hline $30 *$ & 528 & 48 & 48 & 0 \\
\hline 31 & 576 & 384 & 336 & 0 \\
\hline 32 & 192 & 152 & 24 & 0 \\
\hline $33 *$ & 700 & 350 & 90 & 0 \\
\hline $34 *$ & 804 & 412 & 124 & 0 \\
\hline $35 *$ & 714 & 604 & 208 & 0 \\
\hline $36 *$ & 738 & 396 & 56 & 0 \\
\hline $37 *$ & 500 & 296 & 296 & 0 \\
\hline $38 *$ & 366 & 204 & 190 & 0 \\
\hline $39 *$ & 857 & 781 & 553 & 0 \\
\hline 40 & 396 & 250 & 72 & 0 \\
\hline 41 & 712 & 256 & 210 & 0 \\
\hline 42 & 526 & 170 & 170 & 0 \\
\hline
\end{tabular}




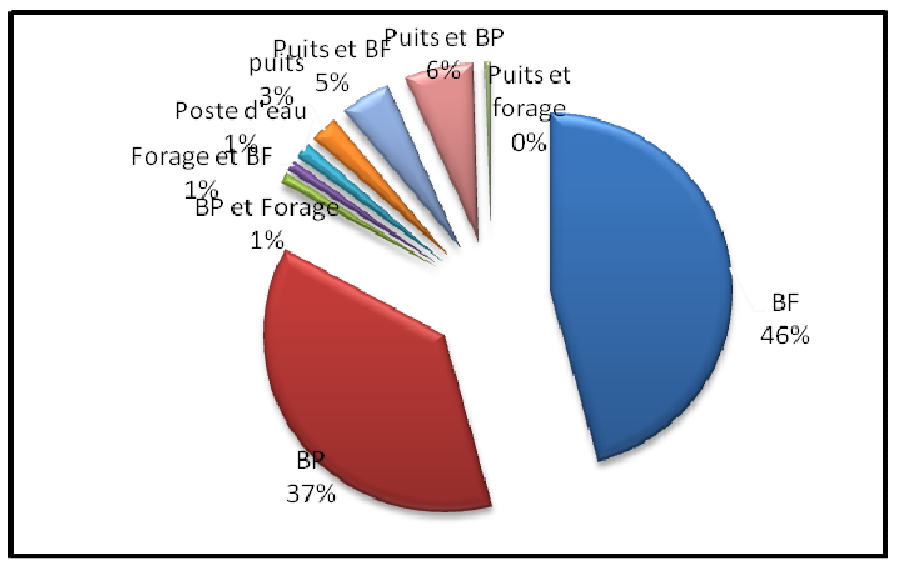

Figure 7 : Mode d'approvisionnment en eau potable.

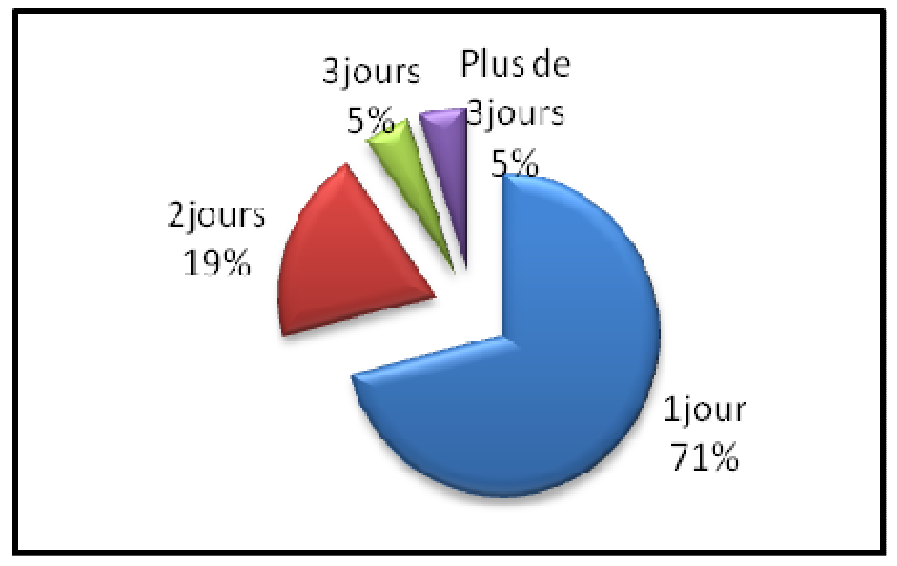

Figure 8 : Durée de stockage de l'eau.

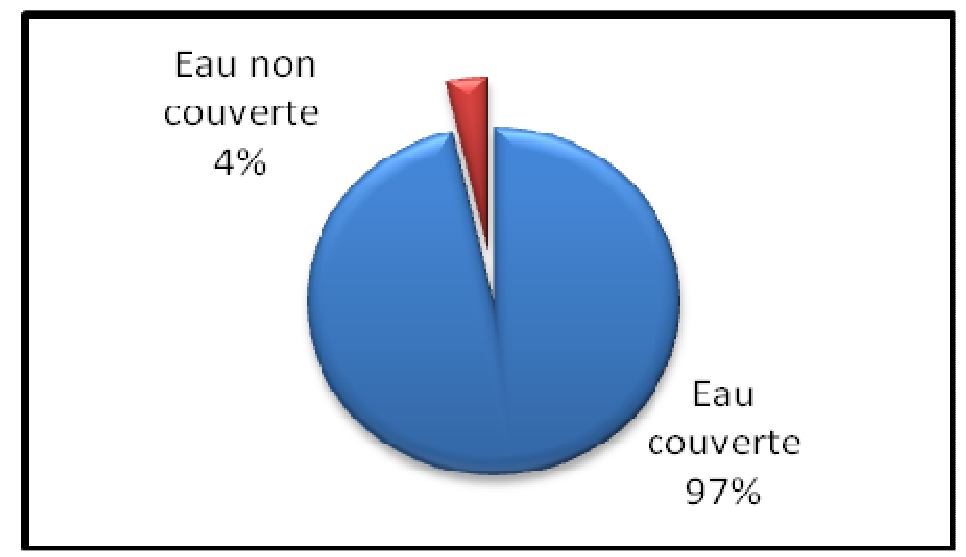

Figure 9: Hygiène liée à la couverture des récipients. 


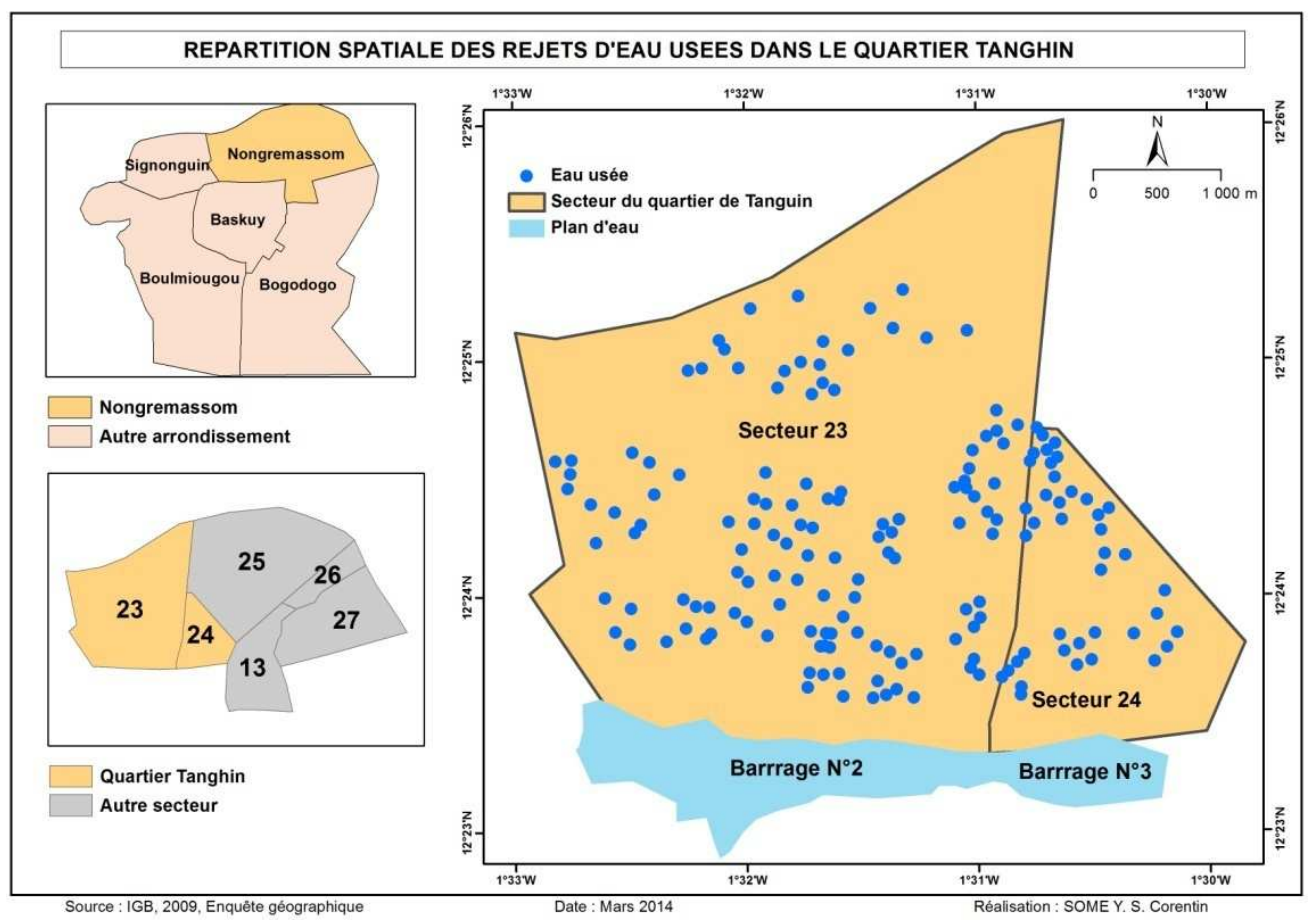

Figure 10: Cartographie des eaux usées dans le quartier Tanghin.

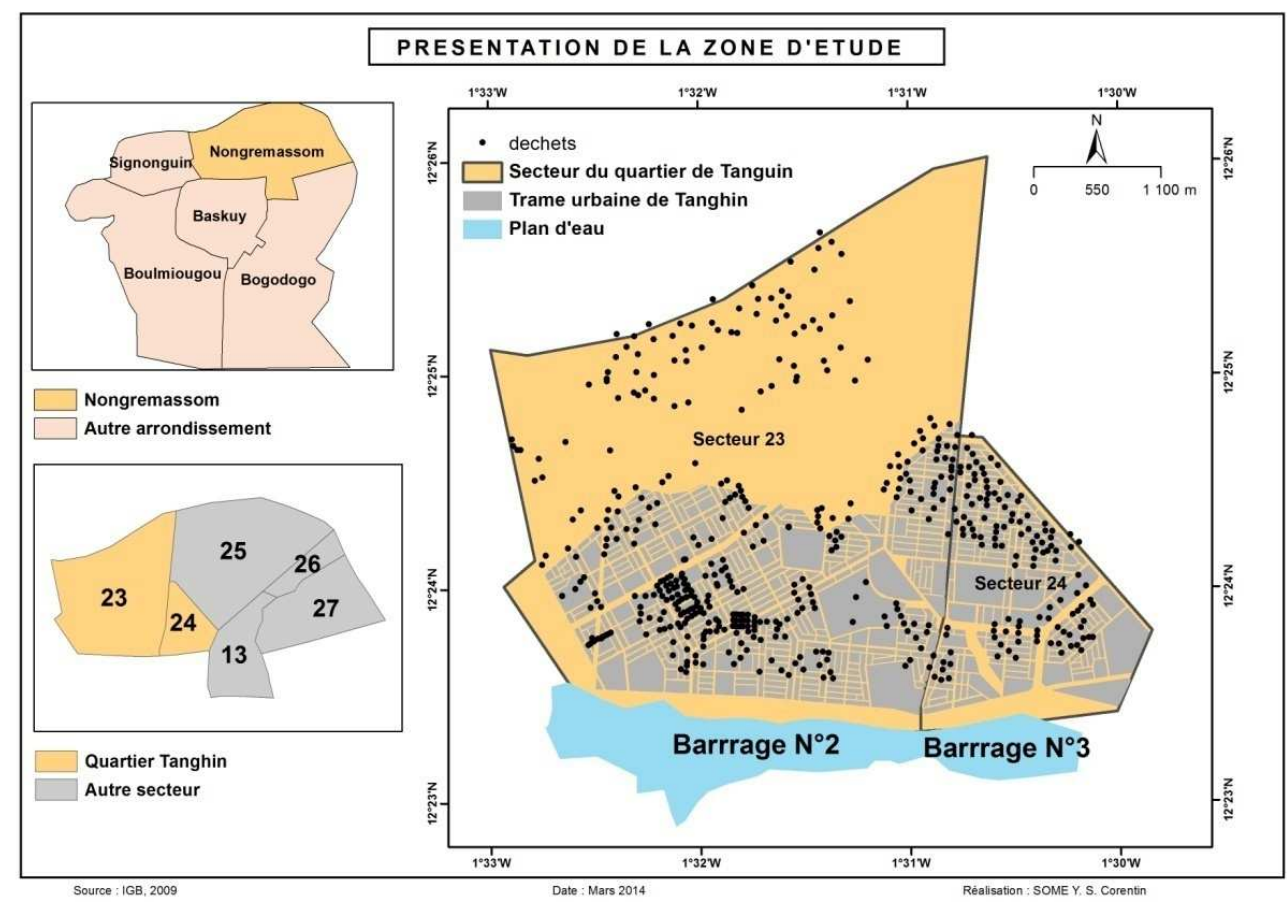

Figure 11: Cartographie des dépotoirs de déchets solides dans le quartier Tanghin. 


\section{DISCUSSION}

Nous remarquons que d'après les résultats, ce sont les maladies transmises par des vecteurs liés à l'eau qui dominent parmi les cas de morbidités des populations. Cela pourrait s'expliquer par le fait de l'absence d'une bonne gestion de l'eau et la carence de mesures de prévention. ce qui contribue à l'accroissement de l'incidence de ces maladies (Cairncross et al., 1980).

$\mathrm{Du}$ point de vue de la gestion de l'assainissement environnemental, le déficit de drainage des quartiers et d'évacuation des eaux usées domestiques observé dans ces milieux précaires est un facteur de maladies, notamment les syndromes pseudo-palustres et diarrhéiques (Dongo et al., 2008).

Les résultats de nos analyses montrent que l'affection la plus répandue est le paludisme. Elle présente une prévalence générale de $8.7 \%$. Ce résultat représente plus du double de celui trouvé par Kafando, (2004) dans le cadre de l'étude épidémiologique dans le quartier de Cissin de la ville de Ouagadougou (3\% de prévalence palustre). Cette prévalence est 8 fois plus élevée que celle évaluée par Dongo et al. (2008) dans le cadre de l'étude épidémiologique menée dans six quartiers précaires à Yopougon. Cette situation s'explique non seulement par le fait que les enquêtes ont été effectuées en saison sèche, mais aussi on pourrait aussi mentionner l'impact du recours à plusieurs moyens de lutte contre les moustiques par les populations (90,1\% des ménages enquêtés utilisent des moustiquaires imprégnées). Cela pourrait s'expliquer aussi par le fait que les zones d'étude diffèrent selon le climat et l'environnement. Cette affection a la plus grande fréquence parmi toutes les affections identifiées. L'étude montre que la tranche d'âge la plus vulnérable aux affections palustres est la tranche d'âge des moins de 5 ans. Ce constat est différent à celui de Kafando (2004) pour qui, la tranche d'âge la plus vulnérable est celle de 5 à 14 ans. Aussi, le genre le plus vulnérable est le genre féminin avec une prévalence de $5.6 \%$ contre
$4.9 \%$ pour le genre masculin. Ce résultat corrobore celui de Kafando (2004).

La diarrhée présente une prévalence générale de $0.36 \%$. Cette valeur est inférieure à celles trouvées par Koné et al. (2007) et Dongo et al. (2008) qui sont respectivement de $7 \%$ et $19 \%$. Cela pourrait s'expliquer par le fait que leur technique des collectes des données et /ou de détection des cas de maladies se faisait par la technique de goutte épaisse encore plus précise que l'enquête de témoignages utilisé dans notre étude. En effet, d'après l'enquête, une très faible proportion de la population utilise l'eau de puits comme boisson $3 \%$ par rapport à l'eau de la borne fontaine et $\mathrm{du}$ robinet particulier dont les proportions sont de $49 \%$ et de $37 \%$ respectivement (Figure 8) ce qui expliquerait la faible prévalence de maladie d'origine hydrique due à l'ingestion d'eau de puits dans le quartier. La tranche la plus vulnérable est celle des moins de cinq ans. Cela est conforme avec de nombreux autres travaux (Who, 1999; Child Health and Development Department, 2002; Who, 2002; Barreto et al., 2006 ; Wierzba et al., 2006; Koné et al., 2007et Dos Santos 2011).

D'après Ngounou (2007), les enquêtes épidémiologiques sont les seuls instruments de mesure en principe objectifs de l'impact de la qualité des eaux sur la population.

La qualité de l'eau joue un rôle important dans l'avènement des maladies hydriques telles que la diarrhée et la fièvre typhoïde. Les eaux des puits ont des charges bactériennes très élevées .Cela serait dû à leur exposition à l'air.

En effet, les résultats d'analyse des eaux de puits montrent qu'aucun puits n'a de l'eau de qualité apte à la consommation selon les normes internationales (Who, 2004). Ces résultats confirment ceux de plusieurs autres études (Makoutode et al., 1999 ; Koné et al., 2007 ; Ndounla, 2007). En ce qui concerne l'utilisation de ces eaux pour l'hygiène individuelle et des ménages, les puits les moins pollués ont des valeurs moyennes de pollution comprises entre 455 et 508 ufc/100 $\mathrm{ml}$ d'eau. Cet intervalle de valeurs correspond 
à $5-10 \%$ de risques de gastro-entérite et à 1.9 - $3.9 \%$ de risques d'infections respiratoires aigües (IRA) fébrile (Who, 2003). En conclusion, les eaux de puits constituent des risques sanitaires pour tout usage domestique.

En ce qui concerne des eaux de boisson stockées au niveau des ménages, les résultats montrent que la qualité de l'eau est modifiée entre la source et la consommation. Ainsi la quasi-totalité (99\%) des eaux de boisson stockées sont polluées. Ces résultats confirment ceux d'Ousseini (2010) menés sur l'accessibilité de l'eau à Tanghin.

Cette pollution pourrait s'expliquer par le mode de collecte et de gestion de l'eau. En effet, $71 \%$ des ménages enquêtés conservent pendant un jour, $19 \%$ pendant deux jours et $6 \%$ des ménages pendant trois et plus de 3 jours. Ces résultats sont comparables à ceux deKombasséré (2007) et Ousseini (2010).

Les résultats de notre enquête qui impliquent la responsabilité de la durée de stockage de l'eau dans la pollution ou la dégradation de l'eau corroborent ceux de Ngnikam et al., (2007) qui a montré que le type de récipient, la durée de stockage de l'eau utilisée pour la conservation de l'eau peut contribuer à la détérioration de la qualité. Ainsi, la mauvaise qualité des eaux de puits et des eaux de boisson stockées de ménages et de l'état environnemental du quartier Tanghin constituent des risques sanitaires pour les populations. C'est en cela que Ledeur (2004) et Ersey (1991) déclarent dans leurs différentes études, que la mauvaise qualité de l'eau, l'hygiène défectueuse et le manque d'assainissement sont à l'origine des maladies hydriques. Pour Kientga (2008), les maladies liées à l'eau telles que le paludisme, le choléra, la diarrhée, la fièvre typhoïde, ont un lien avec les déchets (solides et liquides).

\section{Conclusion}

Le quartier Tanghin, de par sa position géographique aux abords des barrages $\mathrm{N}^{\circ} 2$ et $\mathrm{N}^{\circ} 3$ de Ouagadougou, sa diversité de niveaux d'aménagement, sa diversité socioculturelle, se présente comme un site dans lequel la question de maladies liées peut prendre une importance particulière. $\mathrm{Ce}$ travail de recherche a eu pour objectif d'étudier la contribution des maladies liées à l'eau aux dix principales causes de consultations à Tanghin et les facteurs de risques comportementaux et environnementaux qui y contribuent.

L'analyse de la situation sanitaire du quartier Tanghin dans le tissu urbain de l'arrondissement de Nongr-Masson (Ouagadougou) s'est basée sur les enquêtes géographiques, les enquêtes ménages et l'analyse des statistiques sanitaires du district sanitaire de Kossodo couvrant l'arrondissement de Nongr-Masson.

Cette étude a intégré des données multi-sources comprenant à la fois des informations qualitatives, des informations quantitatives et des informations spatiales. L'étude révèle l'importance des maladies liées à l'eau parmi les principales causes de consultations. Ainsi a-t-il été donné de constater une fréquence importante du paludisme parmi les maladies liées à l'eau les plus récurrentes au niveau des ménages. Cette étude soulève de nombreuses insuffisances dans le système de gestion des déchets solides et liquides avec une importante quantité de dépotoirs sauvages et rejets sauvages d'eaux usées. Ces derniers constituent des facteurs environnementaux importants qui peuvent contribuer à la propagation des pathogènes en leur servant de réservoir. L'action combinée des divers facteurs environnementaux (mauvaise gestion des eaux usées, déchets solides), le mode d'approvisionnement et de gestion de l'eau exposent les populations à des maladies telles que le paludisme et les diarrhées.

\section{REMERCIEMENTS}

Communauté de pratique Ecosanté, Afrique de l'ouest du centre (COPES-AOC) Fondation 2iE Marie de Nongr-Masson.

\section{REFERENCES}

Barreto MLMC. 2006. Community-based monitoring of diarrhea in urban Brazilian Children: Indicence and associated pathogens. Transactions of the Royal 
Society of Tropical Medicine and Hygiene, 100: 234-242.

Cairncross SB. 1980. Evalutation for Village Water Supply Planning. John Wiley \& Sons: Chischester.

Camdessus M, Badré B, Chéret I, TénièreBuchot PF. 2004. Eau. Edition Robert Laffont: Paris. Jeune Afrique Economie 360: $40-46$.

Child Health and Development Department W, 2. 2002. Child Health Epidemiology Reference Group (CHERG), 1-23.

DEP. 2008. Plan d'action du district sanitaire de Nongr-massom. Ministère de la santé publique, Direction des etudes et de la planification, Ouagadougou, 151p

DEP. 2010. Plan d'action du district sanitaire de Nongr-massom. Ministère de la santé publique, Direction des etudes et de la planification, Ouagadougou, 151p

Diakité NR, Adja AM, von Stamm T, Utzinger J, N'Goran EK. 2010. Situation épidémiologique avant la mise en eau du barrage hydroagricole de cinq villages de Bouaké, centre Côte-d'Ivoire. Bulletin de la Société de Pathologie Exotique, 103(1) : 22-28.

Dongo K, Kouamé F, Koné B, Biémi J, Tanner M, Cisssé G. 2008. Analyse de la situation de l'environnement sanitaire des quartiers défavorisés dans le tisssu urabin de Yopougon à Abidjan,Côte d'Ivoire. Vertigo, 8(3): 11.

Dos Santos S. 2011. Les risques sanitaires liés aux usages domestiques de l'eau. Représentations sociales mossi à Ouagadougou (Burkina Faso). Natures Sciences Sociétés, 19(2): 103-112.

Ersey SAJP 1991. Effects of improved water supplyand sanitation on ascariasis, diarrhoea and trochoma. Bulletin of World Health , 609-621.

INSD. 1996. Etat et structure de la population.Rapport final, $41 \mathrm{p}$

Kafando Y. 2004. Environnement urbain et problèmes de santé à Ougadougou :cas du quartier de Cissin. Memoire de maîtrise de Geographie. Université de Ouagadougou, 128p
Kientga S. 2008. Contribution du SIG à l'analyse des leisn déchets-santé en milieu urbain dans les pays en développement. Cas des deux secteurs de la ville de Ouagadougou, Bukina Faso. Thèse de Doctorat, Ecole polytechnique fédérale de Lausanne, 241p.

Kombasséré WK. 2007. L'accès à l'eau potable et les risques diarrhéiques dans les zones irrégulières de Ouagadougou: les cas de Yamtenga. Memoire de Maîtrise de Géographie, Université de Ouagadougou, Ouagadougou, 67p.

Koné B, Doumbia M, Adji FX. 2007. Approche écosystémique à la gestion des maladies diarrhéiques en milieu périurbain : cas d'un village lagunaire dans la commune de yopougon (AbidjanCôte d'ivoire). Rapport final du projet du centre suisse de recherche scientifique, $81 \mathrm{p}$.

Kpoda NW, Sorgho H, Poda JN, Ouédraogo, JB, Kabré GB. 2013. Endémie bilharzienne à Schistosoma mansoni à la vallée du Kou: caractérisation du système de transmission et impact socioéconomique. Comptes Rendus Biologies, 336(5): 284-288.

Ledeur S. 2004. Utilisation d'un système dinformation géographique comme outil de compréhension des épidemies d'origines hydrique et comme outil d'aide à la decision:étude dans un contexte urbain (Ouagadougou, Burkina Faso). Memoire d'ingénieur du Génie sanitaire, Ecole nationale de santé publique (ENSP) Rennes, 65p.

Makoutode MAM. 1999. Qualité et Mode de l'eau des puits en Milieu Rural au Bénin: cas de la sous préfecture de Grand Popo. Medecine d'Afrique Noire, 46(11): 528534.

Ndounla J. 007. Caractéristiques biologiques et physico-chimiques de l'eau de consommation et influence du mode d'approvisionnement sur la santé des populations à Dschang. Rapport de fin de cycle de Master of Sciences, Université de Dschang, 122p. 
Ngnikam EB, Mougoue F, Tie. 2007. Eau, Assainissement et impact sur la santé :étude de cas d'un écosystème urbain à Yaoundé. Actes des JSIRAUF, 89p.

Ngounou NB, Lewa S, Ekodeck GE. 2007. Problématique de L'Accès à L'Eau Potable Dans la Ville de Ngaoundéré (Centre Nord-Cameroun). European Journal of Scientific Research, 18(2): 223-230.

Ouledi A, Toyb M, Aubry P, Gaüzere BA. 2012. Histoire sanitaire et enjeux sanitaires de l'Union des Comores en 2012. Médecine et Santé Tropicales, 22(4): 346-354.

Ousseini. 2010. Analyse spatiale de l'accès à l'eau potable et risques hydriques dans les quartiers péripheriques de Ouagadougou: cas du quartier Tanghin. Memoire de l'obtention du master d'ingenierie de l'eau et de l'environnement, Institut international d'ingénierie de l'eau et de l'environnement, Ouagadougou, 73p.

Poda JN. 2007. Les maladies liées à l'eau dans le bassin de la Volta : état des lieux et perspectives. Volta Basin Focal Project Report No 4. IRD, Montpellier, France, and CPWF, Colombo, Sri Lanka, 87 p.

Resh VH. 2009. Vector-Borne Diseases of Freshwater Habitats, in Encyclopedia of Inland Waters. Academic Press: Oxford; 129-137.

Rodier J. 1996. L'analyse de l'Eau. Dumond : Paris ; 1384p.
Sabatinelli LG. 1989. Paludisme dans la Ville de Ouagadougou. Organisation Mondiale de la Santé ; 7p.

SEMT. 2006. Santé de l'Environnement et du Milieu de Travail. 2006. Rôle des concentrations residuelles de desinfectanr - Eschericjai coli- Recommanadations pour la qualité de l'eau potable au Canada. Consulté en novembre 2006, sur http:// www.hc-sc.gc.ca/ewh-semt/pubw/ water-eau/doc-sup-appuiescherichia_coli/ index_fhtml.

WHO. 1999. The World Health Report 1999: Making a difference.1-136.

WHO. 2002. The World health Report: Reducing Risks, Promoting Healthy Life. 1-230.

WHO. 2003. Guidelines for safe recreational water environments. Coastal and Fresh Waters, 1: 1-253.

WHO. 2004. Guidelines for Drinking-water Quality: Recommandations. WHO, 3(1); 1-542.

Wierzba TF , Abdel-Messih IA , AbuElyazeed R, Putnam SD, Kamal KA, Rozmajzl P, Ahmed SF, Fatah A, Zabedy K, Shaheen HI, Sanders J, Frenck R. 2006. Clinic-based surveillance for bacterial and rotavirus-associated diarrhea in Egyptian children. American Journal of Tropical Medecine and Hygiène, 74: 148-153. 